\title{
CONSECUTIVE PRIMES AND BEATTY SEQUENCES
}

\author{
WILLIAM D. BANKS AND VICTOR Z. GUO
}

Abstract. Fix irrational numbers $\alpha, \hat{\alpha}>1$ of finite type and real numbers $\beta, \hat{\beta} \geqslant 0$, and let $\mathcal{B}$ and $\hat{\mathcal{B}}$ be the Beatty sequences

$$
\mathcal{B}:=(\lfloor\alpha m+\beta\rfloor)_{m \in \mathbb{N}} \quad \text { and } \quad \hat{\mathcal{B}}:=(\lfloor\hat{\alpha} m+\hat{\beta}\rfloor)_{m \in \mathbb{N}} .
$$

In this note, we study the distribution of pairs $\left(p, p^{\sharp}\right)$ of consecutive primes for which $p \in \mathcal{B}$ and $p^{\sharp} \in \hat{\mathcal{B}}$. Under a strong (but widely accepted) form of the Hardy-Littlewood conjectures, we show that

$$
\mid\left\{p \leqslant x: p \in \mathcal{B} \text { and } p^{\sharp} \in \hat{\mathcal{B}}\right\} \mid=(\alpha \hat{\alpha})^{-1} \pi(x)+O\left(x(\log x)^{-3 / 2+\varepsilon}\right) .
$$

MSC Numbers: 11N05; 11B83.

Keywords: primes, Beatty sequence, consecutive, heuristics, HardyLittlewood.

\section{INTRODUCTION}

For any given real numbers $\alpha>0$ and $\beta \geqslant 0$, the associated (generalized) Beatty sequence is defined by

$$
\mathcal{B}_{\alpha, \beta}:=(\lfloor\alpha m+\beta\rfloor)_{m \in \mathbb{N}},
$$

where $\lfloor t\rfloor$ is the largest integer not exceeding $t$. If $\alpha$ is irrational, it follows from a classical exponential sum estimate of Vinogradov [7] that $\mathcal{B}_{\alpha, \beta}$ contains infinitely many prime numbers; in fact, one has

$$
\#\left\{\text { prime } p \leqslant x: p \in \mathcal{B}_{\alpha, \beta}\right\} \sim \alpha^{-1} \pi(x) \quad(x \rightarrow \infty)
$$

where $\pi(x)$ is the prime counting function.

Throughout this paper, we fix two (not necessarily distinct) irrational numbers $\alpha, \hat{\alpha}>1$ and two (not necessarily distinct) real numbers $\beta, \hat{\beta} \geqslant 0$, and we denote

$$
\mathcal{B}:=\mathcal{B}_{\alpha, \beta} \quad \text { and } \quad \hat{\mathcal{B}}:=\mathcal{B}_{\hat{\alpha}, \hat{\beta}} .
$$

Our aim is to study the set of primes $p \in \mathcal{B}$ for which the next larger prime $p^{\sharp}$ lies in $\hat{\mathcal{B}}$. The results we obtain are conditional, relying only on the Hardy-Littlewood conjectures in the following strong form. Let $\mathcal{H}$ be a finite subset of $\mathbb{Z}$, and let $\mathbf{1}_{\mathbb{P}}$ denote the indicator function of the primes. The Hardy-Littlewood conjecture for $\mathcal{H}$ asserts that the estimate

$$
\sum_{n \leqslant x} \prod_{h \in \mathcal{H}} \mathbf{1}_{\mathbb{P}}(n+h)=\mathfrak{S}(\mathcal{H}) \int_{2}^{x} \frac{d u}{(\log u)^{|\mathcal{H}|}}+O\left(x^{1 / 2+\varepsilon}\right)
$$

Date: August 12, 2018. 
holds for any fixed $\varepsilon>0$, where $\mathfrak{S}(\mathcal{H})$ is the singular series given by

$$
\mathfrak{S}(\mathcal{H}):=\prod_{p}\left(1-\frac{|(\mathcal{H} \bmod p)|}{p}\right)\left(1-\frac{1}{p}\right)^{-|\mathcal{H}|} .
$$

Our main result is the following.

THEOREM 1.1. Fix irrational numbers $\alpha, \hat{\alpha}>1$ of finite type and real numbers $\beta, \hat{\beta} \geqslant 0$, and let $\mathcal{B}$ and $\hat{\mathcal{B}}$ be the Beatty sequences given by (1.1). For every prime $p$, let $p^{\sharp}$ denote the next larger prime. Suppose that the Hardy-Littlewood conjecture (1.2) holds for every finite subset $\mathcal{H}$ of $\mathbb{Z}$. Then, for any fixed $\varepsilon>0$, the counting function

$$
\pi(x ; \mathcal{B}, \hat{\mathcal{B}}):=\mid\left\{p \leqslant x: p \in \mathcal{B} \text { and } p^{\sharp} \in \hat{\mathcal{B}}\right\} \mid
$$

satisfies the estimate

$$
\pi(x ; \mathcal{B}, \hat{\mathcal{B}})=(\alpha \hat{\alpha})^{-1} \pi(x)+O\left(x(\log x)^{-3 / 2+\varepsilon}\right),
$$

where the implied constant depends only on $\alpha, \hat{\alpha}$ and $\varepsilon$.

Our results are largely inspired by the recent breakthrough paper of Lemke Oliver and Soundararajan [3], which studies the surprisingly erratic distribution of pairs of consecutive primes amongst the $\phi(q)^{2}$ permissible reduced residue classes modulo $q$. In [3] a conjectural explanation for this phenomenon is given which is based on the strong form of the Hardy-Littlewood conjectures considered in this note, that is, under the hypothesis that the estimate (1.2) holds for every finite subset $\mathcal{H}$ of $\mathbb{Z}$.

\section{Preliminaries}

2.1. Notation. The notation $\llbracket t \rrbracket$ is used to denote the distance from the real number $t$ to the nearest integer; that is,

$$
\llbracket t \rrbracket:=\min _{n \in \mathbb{Z}}|t-n| \quad(t \in \mathbb{R}) .
$$

We denote by $\lfloor t\rfloor$ and $\{t\}$ the greatest integer $\leqslant t$ and the fractional part of $t$, respectively. We also write $\mathbf{e}(t):=e^{2 \pi i t}$ for all $t \in \mathbb{R}$, as usual.

Let $\mathbb{P}$ denote the set of primes in $\mathbb{N}$. In what follows, the letter $p$ always denotes a prime number, and $p^{\sharp}$ is used to denote the smallest prime greater than $p$. In other words, $p$ and $p^{\sharp}$ are consecutive primes with $p^{\sharp}>p$. We also put

$$
\delta_{p}:=p^{\sharp}-p \quad(p \in \mathbb{P}) .
$$

For an arbitrary set $\mathcal{S}$, we use $\mathbf{1}_{\mathcal{S}}$ to denote its indicator function:

$$
\mathbf{1}_{\mathcal{S}}(n):= \begin{cases}1 & \text { if } n \in \mathcal{S} \\ 0 & \text { if } n \notin \mathcal{S} .\end{cases}
$$

Throughout the paper, implied constants in symbols $O, \ll$ and $\gg$ may depend (where obvious) on the parameters $\alpha, \hat{\alpha}, \varepsilon$ but are absolute otherwise. For given functions $F$ and $G$, the notations $F \ll G, G \gg F$ and $F=O(G)$ are all equivalent to the statement that the inequality $|F| \leqslant c|G|$ holds with some constant $c>0$. 
2.2. Discrepancy. We recall that the discrepancy $D(M)$ of a sequence of (not necessarily distinct) real numbers $x_{1}, x_{2}, \ldots, x_{M} \in[0,1)$ is defined by

$$
D(M):=\sup _{\mathcal{I} \subseteq[0,1)}\left|\frac{V(\mathcal{I}, M)}{M}-\right| \mathcal{I}||
$$

where the supremum is taken over all intervals $\mathcal{I}=(b, c)$ contained in $[0,1)$, the quantity $V(\mathcal{I}, M)$ is the number of positive integers $m \leqslant M$ such that $x_{m} \in \mathcal{I}$, and $|\mathcal{I}|=c-b$ is the length of $\mathcal{I}$.

For any irrational number $a$ we define its type $\tau=\tau(a)$ by the relation

$$
\tau:=\sup \left\{t \in \mathbb{R}: \liminf _{n \rightarrow \infty} n^{t} \llbracket a n \rrbracket=0\right\} .
$$

Using Dirichlet's approximation theorem, one sees that $\tau \geqslant 1$ for every irrational number $a$. Thanks to the work of Khinchin $[\mathbf{1}]$ and Roth $[\mathbf{5}, \mathbf{6}]$ it is known that $\tau=1$ for almost all real numbers (in the sense of the Lebesgue measure) and for all irrational algebraic numbers, respectively.

For a given irrational number $a$, it is well known that the sequence of fractional parts $\{a\},\{2 a\},\{3 a\}, \ldots$, is uniformly distributed modulo one (see, for example, [2, Example 2.1, Chapter 1]). When $a$ is of finite type, this statement can be made more precise. By [2, Theorem 3.2, Chapter 2] we have the following result.

Lemma 2.1. Let a be a fixed irrational number of finite type $\tau$. For every $b \in \mathbb{R}$ the discrepancy $D_{a, b}(M)$ of the sequence of fractional parts $(\{a m+b\})_{m=1}^{M}$ satisfies the bound

$$
D_{a, b}(M) \leqslant M^{-1 / \tau+o(1)} \quad(M \rightarrow \infty),
$$

where the function implied by $o(\cdot)$ depends only on a.

2.3. Indicator function of a Beatty sequence. As in $\S 1$ we fix (possibly equal) irrational numbers $\alpha, \hat{\alpha}>1$ and (possibly equal) real numbers $\beta, \hat{\beta} \geqslant 0$, and we set

$$
\mathcal{B}:=\mathcal{B}_{\alpha, \beta} \quad \text { and } \quad \hat{\mathcal{B}}:=\mathcal{B}_{\hat{\alpha}, \hat{\beta}} .
$$

In what follows we denote

$$
a:=\alpha^{-1}, \quad \hat{a}:=\hat{\alpha}^{-1}, \quad b:=\alpha^{-1}(1-\beta) \quad \text { and } \quad \hat{b}:=\hat{\alpha}^{-1}(1-\hat{\beta}) .
$$

It is straightforward to show that

$$
\mathbf{1}_{\mathcal{B}}(m)=\psi_{a}(a m+b) \quad \text { and } \quad \mathbf{1}_{\hat{\mathcal{B}}}(m)=\psi_{\hat{a}}(\hat{a} m+\hat{b}) \quad(m \in \mathbb{N}),
$$

where for any $t \in(0,1)$ we use $\psi_{t}$ to denote the periodic function of period one defined by

$$
\psi_{t}(x):= \begin{cases}1 & \text { if } 0<\{x\} \leqslant t \\ 0 & \text { if } t<\{x\}<1 \text { or }\{x\}=0 .\end{cases}
$$

2.4. Modified Hardy-Littlewood conjecture. For their work on primes in short intervals, Montgomery and Soundararajan [4] have introduced the modified singular series

$$
\mathfrak{S}_{0}(\mathcal{H}):=\sum_{\mathcal{T} \subseteq \mathcal{H}}(-1)^{|\mathcal{H} \backslash \mathcal{T}|} \mathfrak{S}(\mathcal{T})
$$


for which one has the relation

$$
\mathfrak{S}(\mathcal{H})=\sum_{\mathcal{T} \subseteq \mathcal{H}} \mathfrak{S}_{0}(\mathcal{T})
$$

Note that $\mathfrak{S}(\varnothing)=\mathfrak{S}_{0}(\varnothing)=1$. The Hardy-Littlewood conjecture (1.2) can be reformulated in terms of the modified singular series as follows:

$$
\sum_{n \leqslant x} \prod_{h \in \mathcal{H}}\left(\mathbf{1}_{\mathbb{P}}(n+h)-\frac{1}{\log n}\right)=\mathfrak{S}_{0}(\mathcal{H}) \int_{2}^{x} \frac{d u}{(\log u)^{|\mathcal{H}|}}+O\left(x^{1 / 2+\varepsilon}\right) .
$$

LEMMA 2.2. We have

$$
\begin{aligned}
\sum_{1 \leqslant t \leqslant h-1} \mathfrak{S}_{0}(\{0, t\}) & \ll h^{1 / 2+\varepsilon}, \\
\sum_{1 \leqslant t \leqslant h-1} \mathfrak{S}_{0}(\{t, h\}) & \ll h^{1 / 2+\varepsilon}, \\
\sum_{1 \leqslant t_{1}<t_{2} \leqslant h-1} \mathfrak{S}_{0}\left(\left\{t_{1}, t_{2}\right\}\right) & =-\frac{1}{2} h \log h+\frac{1}{2} A h+O\left(h^{1 / 2+\varepsilon}\right),
\end{aligned}
$$

where $A:=2-C_{0}-\log 2 \pi$ and $C_{0}$ denotes the Euler-Mascheroni constant.

Proof. Let us denote

$$
B:=\sum_{1 \leqslant t \leqslant h-1} \mathfrak{S}_{0}(\{0, t\}), \quad C:=\sum_{1 \leqslant t \leqslant h-1} \mathfrak{S}_{0}(\{t, h\}),
$$

and

$$
D_{ \pm}:=\sum_{1 \leqslant t_{1}<t_{2} \leqslant h \pm 1} \mathfrak{S}_{0}\left(\left\{t_{1}, t_{2}\right\}\right)
$$

for either choice of the sign \pm . Clearly,

$$
\mathfrak{S}_{0}(\{0, h\})+B+C+D_{-}=D_{+} \quad \text { and } \quad B=\sum_{1 \leqslant t \leqslant h-1} \mathfrak{S}_{0}(\{0, h-t\})=C .
$$

From [4, Equation (16)] we derive the estimates

$$
D_{ \pm}=-\frac{1}{2} h \log h+\frac{1}{2} A h+O\left(h^{1 / 2+\varepsilon}\right) .
$$

Using the trivial bound $\mathfrak{S}_{0}(\{0, h\}) \ll \log \log h$ and putting everything together, we finish the proof.

2.5. Technical lemmas. Let $\nu(u):=1-1 / \log u$. Note that $\nu(u) \asymp 1$ for $u \geqslant 3$.

LEMMA 2.3. Let $c>0$ be a constant, and suppose that $f$ is a function such that $|f(h)| \leqslant h^{c}$ for all $h \geqslant 1$. Then, uniformly for $3 \leqslant u \leqslant x$ and $\lambda \in \mathbb{R}$ we have

$$
\sum_{\substack{h \leqslant(\log x)^{3} \\ 2 \mid h}} f(h) \nu(u)^{h} \mathbf{e}(\lambda h)=\sum_{\substack{h \geqslant 1 \\ 2 \mid h}} f(h) \nu(u)^{h} \mathbf{e}(\lambda h)+O_{c}\left(x^{-1}\right) .
$$


Proof. Write $\nu(u)^{h}=e^{-h / H}$ with $H:=-(\log \nu(u))^{-1}$. Since $H \leqslant \log u$ for $u \geqslant 3$, for any $h>(\log x)^{3}$ we have $h / H \geqslant h^{2 / 3}$ as $u \leqslant x$; therefore,

$\left|\sum_{\substack{h>(\log x)^{3} \\ 2 \mid h}} f(h) \nu(u)^{h} \mathbf{e}(\lambda h)\right| \leqslant \sum_{h>(\log x)^{3}} h^{c} e^{-h^{2 / 3}} \leqslant x^{-1} \sum_{h>(\log x)^{3}} h^{c} e^{h^{1 / 3}-h^{2 / 3}} \ll_{c} x^{-1}$,

and the result follows.

The next statement is an analogue of [3, Proposition 2.1] and is proved using similar methods.

Lemma 2.4. Fix $\theta \in[0,1]$ and $\vartheta=0$ or 1 . For all $\lambda \in \mathbb{R}$ and $u \geqslant 3$, let

$$
\begin{aligned}
R_{\theta, \vartheta ; \lambda}(u):=\sum_{\substack{h \geqslant 1 \\
2 \mid h}} h^{\theta}(\log h)^{\vartheta} \nu(u)^{h} \mathbf{e}(\lambda h), \\
S_{\lambda}(u):=\sum_{\substack{h \geqslant 1 \\
2 \mid h}} \mathfrak{S}_{0}(\{0, h\}) \nu(u)^{h} \mathbf{e}(\lambda h) .
\end{aligned}
$$

When $\lambda=0$ we have the estimates

$$
\begin{aligned}
R_{\theta, 0 ; 0}(u) & =\frac{1}{2} \Gamma(1+\theta)(\log u)^{1+\theta}+O(1), \\
R_{\theta, 1 ; 0}(u) & =\frac{1}{2}(\log 2) \Gamma(1+\theta)(\log u)^{1+\theta}+O(1), \\
S_{0}(u) & =\frac{1}{2} \log u-\frac{1}{2} \log \log u+O(1) .
\end{aligned}
$$

On the other hand, if $\lambda$ is such that $|\lambda| \geqslant(\log u)^{-1}$, then

$$
\max \left\{\left|R_{\theta, \vartheta ; \lambda}(u)\right|,\left|S_{\lambda}(u)\right|\right\} \ll \lambda^{-4} .
$$

Proof. We adapt the proof of [3, Proposition 2.1]. As in Lemma 2.3 we write $\nu(u)^{h}=e^{-h / H}$ with $H:=-(\log \nu(u))^{-1}$. We simplify the expressions $R_{\theta, \vartheta ; \lambda}(u)$, $S_{\lambda}(u)$ and $T_{\lambda}(u)$ by writing

$$
\nu(u)^{h} \mathbf{e}(\lambda h)=e^{-h / H_{\lambda}} \quad \text { with } \quad H_{\lambda}:=\frac{H}{1-2 \pi i \lambda H} .
$$

Since $\Re\left(h / H_{\lambda}\right)=h / H>0$ for any positive integer $h$, using the Cahen-Mellin integral we have

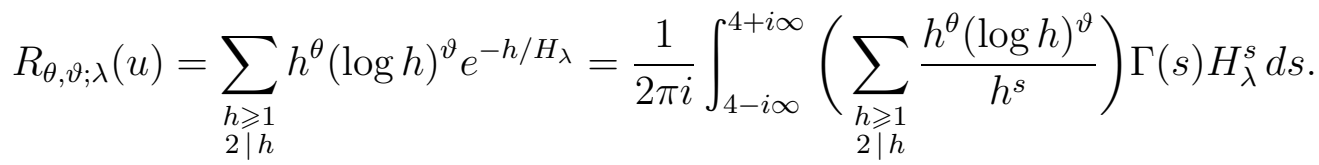

In particular,

$$
R_{\theta, 0 ; \lambda}(u)=\frac{2^{\theta}}{2 \pi i} \int_{4-i \infty}^{4+i \infty} 2^{-s} \zeta(s-\theta) \Gamma(s) H_{\lambda}^{s} d s
$$

and

$$
R_{\theta, 1 ; \lambda}(u)=R_{\theta, 0 ; \lambda}(u) \log 2-\frac{2^{\theta}}{2 \pi i} \int_{4-i \infty}^{4+i \infty} 2^{-s} \zeta^{\prime}(s-\theta) \Gamma(s) H_{\lambda}^{s} d s
$$


When $\lambda \neq 0$ we have

$$
\begin{aligned}
\left|R_{\theta, 0 ; \lambda}(u)\right| & \leqslant \frac{2^{\theta-4}\left|H_{\lambda}\right|^{4}}{2 \pi} \int_{-\infty}^{\infty}|\zeta(4-\theta+i t) \Gamma(4+i t)| d t \\
& \ll\left|H_{\lambda}\right|^{4}=\left(\frac{H^{2}}{1+4 \pi^{2} \lambda^{2} H^{2}}\right)^{2},
\end{aligned}
$$

hence the bound $R_{\theta, 0 ; \lambda}(u) \ll \lambda^{-4}$ holds if $|\lambda| \geqslant(\log u)^{-1}$ since $H \asymp \log u$ for $u \geqslant 3$. In the case that $\lambda=0$, the stated estimate for $R_{\theta, 0 ; 0}(u)$ is obtained by shifting the line of integration in (2.4) to the line $\left\{\Re(s)=-\frac{1}{3}\right\}$ (say), taking into account the residues of the poles of the integrand at $s=1+\theta$ and $s=0$.

Our estimates for $R_{\theta, 1 ; \lambda}(u)$ are proved similarly, using (2.5) instead of (2.4) and taking into account that $\zeta^{\prime}(s-\theta)=(s-1-\theta)^{-1}+O(1)$ for $s$ near $1+\theta$.

Next, for all $\lambda \in \mathbb{R}$ and $u \geqslant 3$, let

$$
T_{\lambda}(u):=\sum_{h \geqslant 1} \mathfrak{S}(\{0, h\}) e^{-h / H_{\lambda}} .
$$

Since $\mathfrak{S}_{0}(\{0, h\})=\mathfrak{S}(\{0, h\})-1$ for all integers $h$, and $\mathfrak{S}(\{0, h\})=0$ if $h$ is odd, it follows that

$$
S_{\lambda}(u)=T_{\lambda}(u)-R_{0,0 ; \lambda}(u)=T_{\lambda}(u)-\frac{1}{2} \log u+O(1) .
$$

Hence, to complete the proof of the lemma, it suffices to show that

$$
T_{0}(u)=\log u-\frac{1}{2} \log \log u+O(1) \quad \text { and } \quad T_{\lambda}(u) \ll \lambda^{-4} \text { if }|\lambda| \geqslant(\log u)^{-1} .
$$

As in the proof of [3, Proposition 2.1], we consider the Dirichlet series

$$
F(s):=\sum_{h \geqslant 1} \frac{\mathfrak{S}(\{0, h\})}{h^{s}}
$$

which can be expressed in the form

$$
F(s)=\frac{\zeta(s) \zeta(s+1)}{\zeta(2 s+2)} \prod_{p}\left(1-\frac{1}{(p-1)^{2}}+\frac{2 p}{(p-1)^{2}\left(p^{s+1}+1\right)}\right),
$$

and the final product is analytic for $\Re(s)>-1$. Using the Cahen-Mellin integral we have

$$
T_{\lambda}(u)=\frac{1}{2 \pi i} \int_{4-i \infty}^{4+i \infty} F(s) \Gamma(s) H_{\lambda}^{s} d s .
$$

For $\lambda \neq 0$ we have

$$
\left|T_{\lambda}(u)\right| \leqslant \frac{\left|H_{\lambda}\right|^{4}}{2 \pi} \int_{-\infty}^{\infty}|F(4+i t) \Gamma(4+i t)| d t \ll\left|H_{\lambda}\right|^{4}=\left(\frac{H^{2}}{1+4 \pi^{2} \lambda^{2} H^{2}}\right)^{2}
$$

hence $T_{\lambda}(u) \ll \lambda^{-4}$ holds provided that $|\lambda| \geqslant(\log u)^{-1}$. For $\lambda=0$, we shift the line of integration in (2.6) to the line $\left\{\Re(s)=-\frac{1}{3}\right\}$ (say), taking into account the double pole at $s=0$ and the simple pole at $s=1$. This leads to the stated estimate for $T_{0}(u)$.

We also need the following integral estimate (proof omitted). 
Lemma 2.5. For all $\lambda \in \mathbb{R}$ and $x \geqslant 3$, let

When $\lambda=0$ we have the estimate

$$
I_{\lambda}(x):=\int_{3}^{x} \frac{\mathbf{e}(\lambda u)}{\nu(u) \log u} d u .
$$

$$
I_{0}(x)=\frac{x}{\log x}+O\left(\frac{x}{(\log x)^{2}}\right)
$$

whereas for any $\lambda \neq 0$ we have

$$
I_{\lambda}(x) \ll|\lambda|^{-1} .
$$

\section{Proof of Theorem 1.1}

For every even integer $h \geqslant 2$ we denote

$\pi_{h}(x ; \mathcal{B}, \hat{\mathcal{B}}):=\mid\left\{p \leqslant x: p \in \mathcal{B}, p^{\sharp} \in \hat{\mathcal{B}}\right.$ and $\left.\delta_{p}=h\right\} \mid=\sum_{n \leqslant x} \mathbf{1}_{\mathcal{B}}(n) \mathbf{1}_{\hat{\mathcal{B}}}(n+h) f_{h}(n)$,

where

$f_{h}(n):=\mathbf{1}_{\mathbb{P}}(n) \mathbf{1}_{\mathbb{P}}(n+h) \prod_{0<t<h}\left(1-\mathbf{1}_{\mathbb{P}}(n+t)\right)= \begin{cases}1 & \text { if } n=p \in \mathbb{P} \text { and } \delta_{p}=h, \\ 0 & \text { otherwise. }\end{cases}$

Clearly,

$$
\pi(x ; \mathcal{B}, \hat{\mathcal{B}})=\sum_{\substack{h \leqslant(\log x)^{3} \\ 2 \mid h}} \pi_{h}(x ; \mathcal{B}, \hat{\mathcal{B}})+O\left(\frac{x}{(\log x)^{3}}\right) .
$$

Fixing an even integer $h \in\left[1,(\log x)^{3}\right]$ for the moment, our initial goal is to express $\pi_{h}(x ; \mathcal{B}, \hat{\mathcal{B}})$ in terms of the function

$$
S_{h}(x):=\sum_{n \leqslant x} f_{h}(n)
$$

recently introduced by Lemke Oliver and Soundararajan [3, Equation (2.5)]. In view of $(2.2)$ we can write

$$
\pi_{h}(x ; \mathcal{B}, \hat{\mathcal{B}})=\sum_{n \leqslant x} \psi_{a}(a n+b) \psi_{\hat{a}}(\hat{a}(n+h)+\hat{b}) f_{h}(n) .
$$

According to a classical result of Vinogradov (see [8, Chapter I, Lemma 12]), for any $\Delta$ such that

$$
0<\Delta<\frac{1}{8} \quad \text { and } \quad \Delta \leqslant \frac{1}{2} \min \{a, 1-a\}
$$

there is a real-valued function $\Psi_{a}$ with the following properties:

(i) $\Psi_{a}$ is periodic with period one;

(ii) $0 \leqslant \Psi_{a}(t) \leqslant 1$ for all $t \in \mathbb{R}$;

(iii) $\Psi_{a}(t)=\psi_{a}(t)$ if $\Delta \leqslant\{t\} \leqslant a-\Delta$ or if $a+\Delta \leqslant\{t\} \leqslant 1-\Delta$;

(iv) $\Psi_{a}$ is represented by a Fourier series

$$
\Psi_{a}(t)=\sum_{k \in \mathbb{Z}} g_{a}(k) \mathbf{e}(k t)
$$

where $g_{a}(0)=a$, and the Fourier coefficients satisfy the uniform bound

$$
\left|g_{a}(k)\right| \ll \min \left\{|k|^{-1},|k|^{-2} \Delta^{-1}\right\} \quad(k \neq 0) .
$$


For convenience, we denote

$$
\mathcal{I}_{a}:=[0, \Delta) \cup(a-\Delta, a+\Delta) \cup(1-\Delta, 1),
$$

so that $\Psi_{a}(t)=\psi_{a}(t)$ whenever $\{t\} \notin \mathcal{I}_{a}$. Defining $\Psi_{\hat{a}}$ and $\mathcal{I}_{\hat{a}}$ similarly with $\hat{a}$ in place of $a$, and taking into account the properties $(i)-($ iii $)$, from (3.2) we deduce that

$$
\pi_{h}(x ; \mathcal{B}, \hat{\mathcal{B}})=\sum_{n \leqslant x} \Psi_{a}(a n+b) \Psi_{\hat{a}}(\hat{a}(n+h)+\hat{b}) f_{h}(n)+O(V(x)),
$$

where $V(x)$ is the number of positive integers $n \leqslant x$ for which

$$
\{a n+b\} \in \mathcal{I}_{a} \quad \text { or } \quad\{\hat{a}(n+h)+\hat{b}\} \in \mathcal{I}_{\hat{a}}
$$

Since $\mathcal{I}_{a}$ and $\mathcal{I}_{\hat{a}}$ are unions of intervals with overall measure $4 \Delta$, it follows from the definition (2.1) and Lemma 2.1 that

$$
V(x) \ll \Delta x+x^{1-1 / \tau+o(1)} \quad(x \rightarrow \infty) .
$$

Now let $K \geqslant \Delta^{-1}$ be a large real number, and let $\Psi_{a, K}$ be the trigonometric polynomial given by

$$
\Psi_{a, K}(t):=\sum_{|k| \leqslant K} g_{a}(k) \mathbf{e}(k t) .
$$

Using (3.3) it is clear that the estimate

$$
\Psi_{a}(t)=\Psi_{a, K}(t)+O\left(K^{-1} \Delta^{-1}\right)
$$

holds uniformly for all $t \in \mathbb{R}$. Defining $\Psi_{\hat{a}, K}$ in a similar way, combining (3.6) with (3.4), and taking into account (3.5), we derive the estimate

$$
\pi_{h}(x ; \mathcal{B}, \hat{\mathcal{B}})=\Sigma_{h}+O\left(\Delta x+x^{1-1 / \tau+\varepsilon}+K^{-1} \Delta^{-1} x\right)
$$

where

$$
\begin{aligned}
\Sigma_{h} & :=\sum_{n \leqslant x} \Psi_{a, K}(a n+b) \Psi_{\hat{a}, K}(\hat{a}(n+h)+\hat{b}) f_{h}(n) \\
& =\sum_{n \leqslant x} \sum_{|k|,|\ell| \leqslant K} g_{a}(k) \mathbf{e}(k(a n+b)) g_{\hat{a}}(\ell) \mathbf{e}(\ell(\hat{a}(n+h)+\hat{b})) f_{h}(n) \\
& =\sum_{|k|,|\ell| \leqslant K} g_{a}(k) \mathbf{e}(k b) g_{\hat{a}}(\ell) \mathbf{e}(\ell \hat{b}) \cdot \mathbf{e}(\ell \hat{a} h) \sum_{n \leqslant x} \mathbf{e}((k a+\ell \hat{a}) n) f_{h}(n) .
\end{aligned}
$$

Therefore

$$
\begin{aligned}
\pi_{h}(x ; \mathcal{B}, \hat{\mathcal{B}})= & \sum_{|k|,|\ell| \leqslant K} g_{a}(k) \mathbf{e}(k b) g_{\hat{a}}(\ell) \mathbf{e}(\ell \hat{b}) \cdot \mathbf{e}(\ell \hat{a} h) \int_{3^{-}}^{x} \mathbf{e}((k a+\ell \hat{a}) u) d\left(S_{h}(u)\right) \\
& +O\left(\Delta x+x^{1-1 / \tau+\varepsilon}+K^{-1} \Delta^{-1} x\right)
\end{aligned}
$$

which completes our initial goal of expressing $\pi_{h}(x ; \mathcal{B}, \hat{\mathcal{B}})$ in terms of the function $S_{h}$. To proceed further, it is useful to recall certain aspects of the analysis of $S_{h}$ 
that is carried out in $[\mathbf{3}]$. First, writing $\widetilde{\mathbf{1}}_{\mathbb{P}}(n):=\mathbf{1}_{\mathbb{P}}(n)-1 / \log n$, up to an error term of size $O\left(x^{1 / 2+\varepsilon}\right)$ the quantity $S_{h}(x)$ is equal to

$$
\begin{aligned}
\sum_{n \leqslant x}\left(\widetilde{\mathbf{1}}_{\mathbb{P}}(n)+\frac{1}{\log n}\right)\left(\widetilde{\mathbf{1}}_{\mathbb{P}}(n+h)+\frac{1}{\log n}\right) \prod_{0<t<h}\left(1-\frac{1}{\log n}-\widetilde{\mathbf{1}}_{\mathbb{P}}(n+t)\right) \\
\quad=\sum_{\mathcal{A} \subseteq\{0, h\}} \sum_{\mathcal{T} \subseteq[1, h-1]}(-1)^{|\mathcal{T}|} \sum_{n \leqslant x}\left(\frac{1}{\log n}\right)^{2-|\mathcal{A}|}\left(1-\frac{1}{\log n}\right)^{h-1-|\mathcal{T}|} \prod_{t \in \mathcal{A} \cup \mathcal{T}} \widetilde{\mathbf{1}}_{\mathbb{P}}(n+t) ;
\end{aligned}
$$

see [3, Equations (2.5) and (2.6)]. By the modified Hardy-Littlewood conjecture (2.3) the estimate

$$
\begin{aligned}
\sum_{n \leqslant x}(\log n)^{-c} \prod_{t \in \mathcal{H}} \widetilde{\mathbf{1}}_{\mathbb{P}}(n+t) & =\int_{3^{-}}^{x}(\log u)^{-c} d\left(\sum_{n \leqslant u} \prod_{t \in \mathcal{H}} \widetilde{\mathbf{1}}_{\mathbb{P}}(n+t)\right) \\
& =\mathfrak{S}_{0}(\mathcal{H}) \int_{3}^{x}(\log u)^{-c-|\mathcal{H}|} d u+O\left(x^{1 / 2+\varepsilon}\right)
\end{aligned}
$$

holds uniformly for any constant $c>0$; consequently, up to an error term of size $O\left(x^{1 / 2+\varepsilon}\right)$ the quantity $S_{h}(x)$ is equal to

$$
\sum_{\mathcal{A} \subseteq\{0, h\}} \sum_{\mathcal{T} \subseteq[1, h-1]}(-1)^{|\mathcal{T}|} \mathfrak{S}_{0}(\mathcal{A} \cup \mathcal{T}) \int_{3}^{x}(\log u)^{-2-|\mathcal{T}|} \nu(u)^{h-1-|\mathcal{T}|} d u
$$

where

$$
\nu(u):=1-\frac{1}{\log u} \quad(u>1)
$$

(note that $\nu(u)$ is the same as $\alpha(u)$ in the notation of $[\mathbf{3}]$ ). For every integer $L \geqslant 0$ we denote

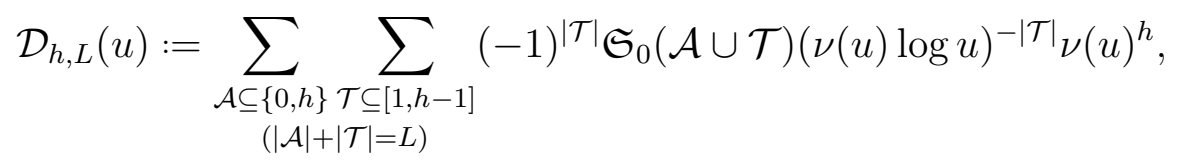

so that

$$
S_{h}(x)=\sum_{L=0}^{h+1} \int_{3}^{x} \nu(u)^{-1}(\log u)^{-2} \mathcal{D}_{h, L}(u) d u+O\left(x^{1 / 2+\varepsilon}\right) .
$$

We now combine this relation with (3.7), sum over the even natural numbers $h \leqslant(\log x)^{3}$, and apply $(3.1)$ to deduce that the quantity $\pi(x ; \mathcal{B}, \hat{\mathcal{B}})$ is equal to

$$
\sum_{\substack{h \leqslant(\log x)^{3} \\ 2 \mid h}} \sum_{L=0}^{h+1} \sum_{\substack{|k|,|\ell| \leqslant K\\}} g_{a}(k) \mathbf{e}(k b) g_{\hat{a}}(\ell) \mathbf{e}(\ell \hat{b}) \cdot \mathbf{e}(\ell \hat{a} h) \int_{3}^{x} \frac{\mathbf{e}((k a+\ell \hat{a}) u)}{\nu(u)(\log u)^{2}} \mathcal{D}_{h, L}(u) d u
$$

up to an error term of size

$$
\ll \frac{x}{(\log x)^{3}}+\left(\Delta x+x^{1-1 / \tau+\varepsilon}+K^{-1} \Delta^{-1} x\right)(\log x)^{3} .
$$

Choosing $\Delta:=(\log x)^{-6}$ and $K:=(\log x)^{12}$ the combined error is $O\left(x /(\log x)^{3}\right)$, which is acceptable. 
Next, arguing as in $[\mathbf{3}]$ and noting that

$$
\sum_{|k|,|\ell| \leqslant K}\left|g_{a}(k) g_{\hat{a}}(\ell)\right| \ll(\log \log x)^{2}
$$

one sees that the contribution to $\pi(x ; \mathcal{B}, \hat{\mathcal{B}})$ coming from terms with $L \geqslant 3$ does not exceed $O\left(x /(\log x)^{5 / 2}\right)$. Since $\mathcal{D}_{h, 1}$ is identically zero (as $\mathfrak{S}_{0}$ vanishes on singleton sets), this leaves only the terms with $L=0$ or $L=2$. The function $\mathcal{D}_{h, 2}$ splits naturally into four pieces according to whether $\mathcal{A}=\varnothing,\{0\},\{h\}$ or $\{0, h\}$. Consequently, up to $O\left(x /(\log x)^{5 / 2}\right)$ we can express the quantity $\pi(x ; \mathcal{B}, \hat{\mathcal{B}})$ as

$$
\sum_{j=1}^{5} \sum_{|k|,|\ell| \leqslant K} g_{a}(k) \mathbf{e}(k b) g_{\hat{a}}(\ell) \mathbf{e}(\ell \hat{b}) \int_{3}^{x} \frac{\mathbf{e}((k a+\ell \hat{a}) u)}{\nu(u)(\log u)^{2}} \mathcal{F}_{j, \ell}(u) d u
$$

where (taking into account Lemma 2.3) we have written

$$
\sum_{\substack{h \leqslant(\log x)^{3} \\ 2 \mid h}} \mathbf{e}(\ell \hat{a} h) \mathcal{D}_{h, L}(u)=\sum_{j=1}^{5} \mathcal{F}_{j, \ell}(u)+O\left(x^{-1}\right)
$$

with

$$
\begin{aligned}
& \mathcal{F}_{1, \ell}(u):=\sum_{\substack{h \geqslant 1 \\
2 \mid h}} \nu(u)^{h} \mathbf{e}(\ell \hat{a} h), \\
& \mathcal{F}_{2, \ell}(u):=\sum_{\substack{h \geqslant 1 \\
2 \mid h}} \mathfrak{S}_{0}(\{0, h\}) \nu(u)^{h} \mathbf{e}(\ell \hat{a} h), \\
& \mathcal{F}_{3, \ell}(u):=\frac{(-1)}{\nu(u) \log u} \sum_{\substack{h \geqslant 1 \\
2 \mid h}} \sum_{\substack{1 \leqslant t \leqslant h-1 \\
\mathfrak{S}_{0}}} \mathfrak{S}_{0}(\{0, t\}) \nu(u)^{h} \mathbf{e}(\ell \hat{a} h), \\
& \mathcal{F}_{4, \ell}(u):=\frac{(-1)}{\nu(u) \log u} \sum_{\substack{h \geqslant 1 \\
2 \mid h}} \sum_{\substack{1 \leqslant t \leqslant h-1\\
}} \mathfrak{S}_{0}(\{t, h\}) \nu(u)^{h} \mathbf{e}(\ell \hat{a} h), \\
& \mathcal{F}_{5, \ell}(u):=\frac{1}{(\nu(u) \log u)^{2}} \sum_{\substack{h \geqslant 1 \\
2 \mid h}} \sum_{1 \leqslant t_{1}<t_{2} \leqslant h-1} \mathfrak{S}_{0}\left(\left\{t_{1}, t_{2}\right\}\right) \nu(u)^{h} \mathbf{e}(\ell \hat{a} h) .
\end{aligned}
$$

First, we show that certain terms in (3.8) make a negligible contribution that does not exceed $O\left(x /(\log x)^{3 / 2-\varepsilon}\right)$.

For any $\ell \neq 0$, using Lemma 2.4 with $\lambda=\ell \hat{a}$ we have

$$
\mathcal{F}_{1, \ell}(u)=R_{0,0 ; \ell \hat{a}}(u) \ll \ell^{-4}
$$

provided that $|\ell \hat{a}| \geqslant(\log u)^{-1}$, and for this it suffices that $u \geqslant \exp (\hat{\alpha})$. Thus,

$$
\int_{3}^{x} \frac{\mathbf{e}((k a+\ell \hat{a}) u)}{\nu(u)(\log u)^{2}} \mathcal{F}_{1, \ell}(u) d u \ll 1+\ell^{-4} \frac{x}{(\log x)^{2}} .
$$


In view of (3.3), the contribution to (3.8) from terms with $j=1$ and $\ell \neq 0$ is

$$
\ll \sum_{\substack{|k|,|\ell| \leqslant K \\ \ell \neq 0}}\left|g_{a}(k)\right| \cdot|\ell|^{-1}\left(1+\ell^{-4} \frac{x}{(\log x)^{2}}\right) \ll \frac{x \log \log x}{(\log x)^{2}} \ll \frac{x}{(\log x)^{3 / 2-\varepsilon}} .
$$

Similarly, for $\ell \neq 0$ and $u \geqslant \exp (\hat{\alpha})$ we have $\mathcal{F}_{2, \ell}(u)=S_{\ell \hat{a}}(u) \ll \ell^{-4}$ by Lemma 2.4, so the contribution to (3.8) from terms with $j=2$ and $\ell \neq 0$ is also $O\left(x /(\log x)^{3 / 2-\varepsilon}\right)$.

For any $\ell \in \mathbb{Z}$, by Lemma 2.2 and Lemma 2.4 we have

$$
\max \left\{\left|\mathcal{F}_{3, \ell}(u)\right|,\left|\mathcal{F}_{4, \ell}(u)\right|\right\} \ll \frac{1}{\log u} \sum_{\substack{h \geqslant 1 \\ 2 \mid h}} h^{1 / 2+\varepsilon / 2} \nu(u)^{h} \ll(\log u)^{1 / 2+\varepsilon / 2},
$$

hence for $j=3,4$ we see that

$$
\int_{3}^{x} \frac{\mathbf{e}((k a+\ell \hat{a}) u)}{\nu(u)(\log u)^{2}} \mathcal{F}_{j, \ell}(u) d u \ll \frac{x}{(\log x)^{3 / 2-\varepsilon / 2}} .
$$

By (3.3), it follows that the contribution to (3.8) from terms with $j=3,4$ is

$$
\ll \frac{x}{(\log x)^{3 / 2-\varepsilon / 2}} \sum_{|k|,|\ell| \leqslant K}\left|g_{a}(k) g_{\hat{a}}(\ell)\right| \ll \frac{x(\log \log x)^{2}}{(\log x)^{3 / 2-\varepsilon / 2}} \ll \frac{x}{(\log x)^{3 / 2-\varepsilon}} .
$$

Finally, for any $\ell \in \mathbb{Z}$ and $u \geqslant \exp (\hat{\alpha})$, by Lemma 2.2 and Lemma 2.4 we have

$$
\begin{aligned}
\mathcal{F}_{5, \ell}(u) & =\frac{1}{(\nu(u) \log u)^{2}} \sum_{\substack{h \geqslant 1 \\
2 \nmid h}}\left(-\frac{1}{2} h \log h+\frac{1}{2} A h+O\left(h^{1 / 2+\varepsilon / 2}\right)\right) \nu(u)^{h} \mathbf{e}(\ell \hat{a} h) \\
& =\frac{-\frac{1}{2} R_{1,1 ; \ell \hat{a}}(u)+\frac{1}{2} A R_{1,0 ; \ell \hat{a}}(u)+O\left(R_{1 / 2+\varepsilon / 2,0 ; 0}(u)\right)}{(\nu(u) \log u)^{2}} \\
& \ll \frac{\lambda^{-4}+(\log u)^{3 / 2+\varepsilon / 2}}{(\log u)^{2}},
\end{aligned}
$$

and arguing as before we see that the contribution to (3.8) coming from terms with $j=5$ does not exceed $O\left(x /(\log x)^{3 / 2-\varepsilon}\right)$.

Applying the preceding bounds to (3.8) we see that, up to $O\left(x /(\log x)^{3 / 2-\varepsilon}\right)$, the quantity $\pi(x ; \mathcal{B}, \hat{\mathcal{B}})$ is equal to

$$
\hat{a} \sum_{j=1,2} \sum_{|k| \leqslant K} g_{a}(k) \mathbf{e}(k b) \int_{3}^{x} \frac{\mathbf{e}(k a u)}{\nu(u)(\log u)^{2}} \mathcal{F}_{j, 0}(u) d u,
$$

where we have used the fact that $g_{\hat{a}}(0)=\hat{a}$. By Lemma 2.4 we have

$$
\mathcal{F}_{1,0}(u)=\sum_{\substack{h \geqslant 1 \\ 2 \nmid h}} \nu(u)^{h}=R_{0,0 ; 0}(u)=\frac{1}{2} \log u+O(1)
$$

and

$$
\mathcal{F}_{2,0}(u)=\sum_{\substack{h \geqslant 1 \\ 2 \mid h}} \mathfrak{S}_{0}(\{0, h\}) \nu(u)^{h}=S_{0}(u)=\frac{1}{2} \log u-\frac{1}{2} \log \log u+O(1) ;
$$


therefore,

$$
\int_{3}^{x} \frac{\mathbf{e}(k a u)}{\nu(u)(\log u)^{2}} \mathcal{F}_{j, 0}(u) d u=\frac{1}{2} \int_{3}^{x} \frac{\mathbf{e}(k a u)}{\nu(u) \log u} d u+O\left(\frac{x \log \log x}{(\log x)^{2}}\right) \quad(j=1,2) .
$$

Consequently, up to $O\left(x /(\log x)^{3 / 2-\varepsilon}\right)$ we can express the quantity $\pi(x ; \mathcal{B}, \hat{\mathcal{B}})$ as

$$
\hat{a} \sum_{|k| \leqslant K} g_{a}(k) \mathbf{e}(k b) \int_{3}^{x} \frac{\mathbf{e}(k a u)}{\nu(u) \log u} d u .
$$

To complete the proof of Theorem 1.1, we apply Lemma 2.5, which shows that the term $k=0$ in (3.9) contributes

$$
a \hat{a} \frac{x}{\log x}+O\left(\frac{x}{(\log x)^{2}}\right)=(\alpha \hat{\alpha})^{-1} \pi(x)+O\left(\frac{x}{(\log x)^{2}}\right)
$$

to the quantity $\pi(x ; \mathcal{B}, \hat{\mathcal{B}})$ (and thus accounts for the main term), whereas the terms in (3.9) with $k \neq 0$ contribute altogether only a bounded amount.

Acknowledgement. The first author was supported in part by a grant from the University of Missouri Research Board.

\section{REFERENCES}

[1] A. Khinchin, Zur metrischen Theorie der diophantischen Approximationen. Math. Z. 24 (1926), no. 4, 706-714.

[2] L. Kuipers and H. Niederreiter, Uniform distribution of sequences. Pure and Applied Mathematics. Wiley-Interscience, New York-London-Sydney, 1974.

[3] R. J. Lemke Oliver and K. Soundararajan, Unexpected biases in the distribution of consecutive primes. Proc. Natl. Acad. Sci. USA, to appear. arXiv:1603.03720

[4] H. L. Montgomery and K. Soundararajan, Primes in short intervals. Comm. Math. Phys. 252 (2004), no. 1-3, 589-617.

[5] K. Roth, Rational approximations to algebraic numbers. Mathematika 2 (1955), 1-20.

[6] K. Roth, Corrigendum to "Rational approximations to algebraic numbers." Mathematika 2 (1955), 168.

[7] I. M. Vinogradov, A new estimate of a certain sum containing primes (Russian). Rec. Math. Moscou, n. Ser. 2(44) (1937), no. 5, 783-792. English translation: New estimations of trigonometrical sums containing primes. C. R. (Dokl.) Acad. Sci. URSS, n. Ser. 17 (1937), 165-166.

[8] I. M. Vinogradov, The method of trigonometrical sums in the theory of numbers. Dover Publications, Inc., Mineola, NY, 2004.

Department of Mathematics, University of Missouri, Columbia mo, USA.

E-mail address: bankswd@missouri.edu

Department of Mathematics, University of Missouri, Columbia mo, USA.

E-mail address: zgbmf@mail.missouri.edu 